\title{
Multi-Control-Volume Analysis of the Compression Process in a High-Temperature Root's Type Compressor
}

\author{
David I. Blekhman \\ Padnos School of Engineering, Grand Valley State University, Grand Rapids, Michigan, USA \\ Joseph C. Mollendorf, James D. Felske, and John A. Lordi \\ Mechanical and Aerospace Engineering Department, State University of New York at Buffalo, \\ Buffalo, New York, USA
}

In this study we have modeled a high-temperature Root's type compressor and compared predicted results with measurements. Compression process is modeled using multiple control volumes. Initially, there are two control volumes connected by an "expansion nozzle" naturally formed by the rotor tip and the blower casing. The "nozzle" has time-varying geometry and is modeled to allow transition from choked to non-choked flow. The well-filling process is modeled via expansion of the compressed gas from the high-pressure reservoir into the well through this "nozzle." When the pressures in the well and reservoir equalize, the "passive" part of the compression process is taken to be complete. At this point, the gas in the well is assumed to be fully-mixed and the two control volumes are subsequently considered as one. Then the "active" part of compression takes place. Numerical computations are shown to agree well with the experimental data.

Keywords Compressor, Heater, Pump, Pyrolitic, Roots, Supercharger

Design of a Root's type machine for the production of a high temperature stream of gas can hardly rely on the simple performance expressions introduced early in the twentieth century

Received 25 June 2002; accepted 1 July 2002.

The authors gratefully acknowledge the following: Calspan UB Research Center (CUBRC) for grant support of this research; Tom Tressler and David Hokey of DMD-Roots division of Dresser Equipment Group, Inc. for helpful information and sponsorship; Steve Schiffhauer of National Instruments Corporation for valuable advice on the experiment data acquisition set up.

Address correspondence to David I. Blekhman, Padnos School of Engineering, Grand Valley State University, 301 W. Fulton St., Grand Rapids, MI 49504-6431, USA. E-mail: blekhmad@gvsu.edu (e.g., Ware and Wilson, 1927). In this work, departure of the experimental data from assumed polytropic behavior was adjusted by a multiplicative coefficient. The coefficient also varied with the rotational speed of the supercharger. Such an approach was considered as acceptable because the lower the pressure ratio the less error realized. The common use of the ideal Root's blower formula (Taylor, 1966) for calculating the shaft work required for raising the pressure due to the decreasing volume, rapidly losses accuracy as the pressure ratio increases. The complex pressure history in the compressor along with increased leakage, and other factors largely contribute to the deviation.

A significant contribution to the compression theory of a Root's type blower was made by dividing the compression process into two stages (Kestin and Owczarek, 1952). The first stage is an instant expansion of the reservoir gas into the well, where it mixes with the gas in the well thereby raising the pressure and temperature. In the second stage, the combined volume undergoes compression by reduction in volume at constant mass. Once the compression is completed, a certain amount of mass is released through a valve to complete the cycle. This work revealed a very interesting discussion of the effect that the volumetric ratio of the reservoir to the well has on overall performance. We found a closed form solution (Blekhman et al., 2000) to this problem even while gas is allowed to leave the system during the compression stage.

The work of (Patterson and Ritchie, 1969) was the first attempt to describe comprehensively the performance of a Root's blower. The authors included such effects as heat transfer, friction losses and leakage. Unfortunately, the analytical expressions did not include the expansion mixing process. Therefore the finite time of mixing was ignored and, consequently, some reduction in the volume compressed was not included. This resulted in theoretical overestimates of the power required at high speeds and at pressure ratios up to 1.6. 
Very high rates of volumetric displacement and low frictional losses make a Roots type blower almost an ideal supercharger. Many researchers have worked on improving the performance of such superchargers. Behavior of the control volumes and areas involved (Ucer and Celik, 1980) were detailed in a kinematic analysis. Their mathematical model was found to be in good agreement with the available test data. A very comprehensive study (Sorenson, 1984) detailed the governing equations for a particular three-lobe supercharger. A dimensionless flow coefficient was used to adjust the flow rates. A bond graph method was used in a more recent simulation (Merala et al., 1998). Even neglecting heat transfer, the numerical results were found to be in good agreement with measured data. Pressure ratios of up to 1.8 only were reported, probably due to inherent practical limitations on a supercharger.

Much higher pressure ratios are required in analyzing a multirecompression heater (MRH). The concept of MRH was first introduced by (Weatherston, 1969) for use in a large hypersonic testing facility. A rotary mechanical device for achieving high temperatures was described and analyzed. The MRH concept was further developed (Russo and Daiber, 1976) and (Dubrin and Harri, 1979) for an isotope separation process. This idea evolved into a large, complex, four-lobe, internally cooled Root's type MRH. It was intended to reach temperatures of up to $5000 \mathrm{~K}$. However, due to an unanticipated torsional effect in the drivetrain, the prototype, built under the sponsorship of LLNL, failed during no load testing. The probable failure mechanism has been identified (Russo, 1999) and can be properly addressed in future designs. Availability of new high temperature materials may significantly reduce the need for cooling the rotors with adiabatic operation potentially achievable for applications of up to $2000 \mathrm{~K}$.

The continuing need for high temperature sources of contamination free gas combined with new material technologies has refueled interest in MRH. We see MRH potential in applications such as high temperature experimental studies, optical fiber production, and chemical synthesis. Use of an MRH for ethylene production was recently evaluated (Smith, 2001). The study showed that an MRH based process can be a viable alternative in ethylene synthesis.

The present research focuses on a Root's type compressor design as the description of the MRH process. A set of dimensionless gasdynamic equations is developed and analyzed describing processes and conditions for the range of design parameters associated with high output temperatures.

\section{ANALYSIS}

Although many alterations to the Root's blower design have been introduced throughout the years, the basic configuration has stayed the same. Two intermeshing impellers, which are mounted on parallel shafts, are enclosed in a housing, Figure 1. Fluid enters and exits perpendicularly to the plane of the impellers' shafts through inlet and outlet ports. The most common configuration of the Root's type compressor uses two lobe im-

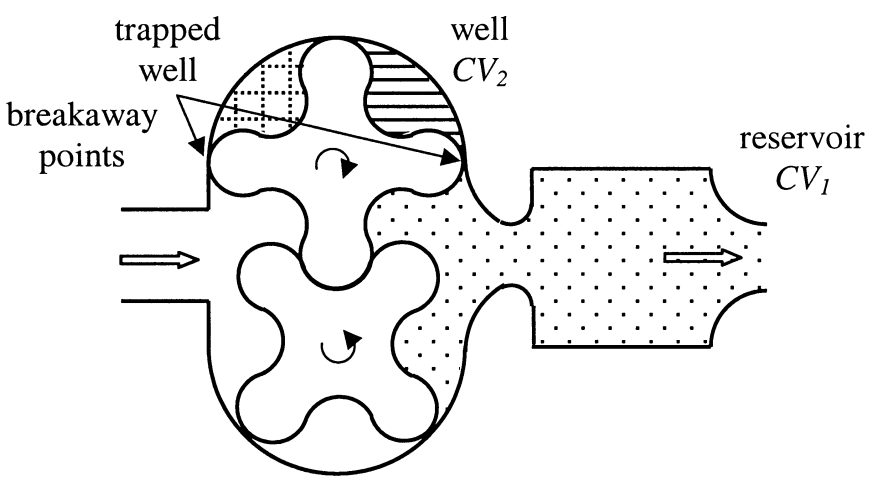

\section{FIGURE 1}

Four-lobe Root's type compressor, showing the position of the impellers an instant before the expansion-mixing process between the reservoir and well.

pellers; three lobe impellers are used less frequently. A four lobe design, considered so far only theoretically, has a lower volumetric displacement than its two lobe counterpart of the same diameter and type of geometry. However, it offers a greater rigidity and reduces the magnitude of the pulsations in the reservoir (Russo, 1999). The lobes' tips and the sides of the impellers are separated from the inner surface of the casing by small gaps (clearances). The clearances are varied in different locations of the compressor in order to accommodate for mechanical and thermal loads. The gap provides the oil-free operation of the compressor. Thus, there is no contamination introduced to the pumped gas and the friction losses are greatly reduced.

A well (CV2) is formed between the casing and two consecutive lobes on an impeller when the second of the two lobes in the direction of the rotation, passes the inlet's breakaway point. This is the point, after passing which, the communication (except for the leakage) between the well and the inlet can be considered to be over. Gas at the "inlet" temperature and pressure is trapped in the well volume. The mechanical action of the rotating well brings the trapped gas to the outlet region. While in the process of moving, the well is in constant communication with the other regions of the blower through the leakage passages. The gas leaking into the well increases the pressure, temperature and mass of the gas in the well, but not to the level of the outlet reservoir. Some of the gas leaks out of the well volume into the lower pressure side.

As the tip of leading lobe reaches the breakaway point of the high pressure reservoir (CV1) the expansion process begins. The reservoir breakaway point is considered to be the location where the minimum distance between the lobe tip and the casing starts to increase sharply from its clearance value due to the rotation of the impeller. Thus, a passage (nozzle) for flow of the high pressure gas is formed. The configuration of the passage constantly changes with the rotation of the lobe. This time dependent behavior is based on the lobe and casing geometries and is unique for each design. Treatment of a particular nozzle shape will be considered in this paper. 
Formation of a nozzle provides a means for the high pressure gas to flow into the well at rates much higher than the leakage. In the high temperature Root's compressor it is expected that the pressure ratio is high enough for choked flow to develop at the beginning of the expansion-mixing process. The reservoir gas flows into the well, where it expands and irreversibly mixes with the well gas. Simultaneously, the well volume slightly decreases, additionally contributing to the compression of the gas in the well. This decrease in volume comes from the propagation of the nozzle throat into the well region as the well rotates toward the nozzle throat. These processes cause the pressure to first increase to the point where the flow through the nozzle becomes unchoked and then to increase until the pressure in the well and reservoir are practically equal. When this occurs, the mixing process is considered finished.

In the next step, the well and the reservoir are taken to be a single control volume (CV3) which undergoes a compression as the remaining portion of the well continues to decrease in volume. After the mixing, the temperatures in both volumes slightly differ. In order to proceed further, a mean temperature of the combined volume is used. The final compression was already discussed in great detail in our previous study (Blekhman et al., 2000). An analytical solution for this part of the problem exists if the flow through the outlet is both adiabatic and choked at all times. Compression ends when a new well is introduced by the other impeller on the opposite side of the high pressure reservoir. The total time for a single combination of expansion-mixing and compression is called the time of compression. The number of compressions per full revolution of the drive shaft is twice the number of lobes per impeller.

A complex process takes place in the reservoir region as well. First, the high pressure gas flows into the well. The pressure fluctuation here depends mainly on the volumetric ratio of the reservoir to the well. The larger the ratio the smaller the fluctuations at a given pressure ratio. Second, the compressed high temperature gas flows out of the device through its outlet port. In contrast to the nozzle flow which exists only as long as the expansion-mixing process takes place, the outlet flow is always present. However, the flow rate is not constant: it pulsates due to the pressure fluctuations. In order to accommodate different down-stream applications the outlet can have different designs-a converging or a converging-diverging nozzle, for example. Depending on the outlet configuration, the down-stream pressure and the operational conditions of the compressor, the outflow could be either choked, unchoked or alternating between these conditions. Third, the reservoir volume has a complex time history. There is a region of the reservoir where volume stays the same. However, in the region, swept by the lobes, the volume continually changes. While one of the wells is undergoing the expansion-mixing process, its leading lobe intrudes into the free space of the reservoir, decreasing the reservoir volume. At the same time on the opposite side of the reservoir, the trailing lobe of a well has just finished its compression and is becoming a moving region of the reservoir. Calculations and (Patterson and
Ritchie, 1969) show that the reservoir volume varies approximately linearly with time; when the mixing process ends the combined control volume becomes a sum of the reservoir and well volumes. The well volume during mixing can be approximated as constant; consequently, the combined volume continues to decrease linearly.

The physical model employs the following assumptions: the working fluid is a perfect gas and its potential and kinetic energies are neglected; the processes are quasi-steady; mixing is instantaneous, and, thus, homogeneous conditions throughout the control-volumes always exist; there is no control volume associated with the "nozzle" because it serves the purpose of quantifying the flow from the reservoir into the well; the inlet is a large reservoir of uniform pressure; and, the viscous and shaft works are taken to be zero.

The first law, mass conservation, and ideal gas equations for the $i$-th $\mathrm{CV}$ are:

$$
\begin{aligned}
\dot{Q}_{i}-P_{i} \frac{d V_{i}}{d t} & =\frac{d\left(M_{i} U_{i}\right)}{d t}+\dot{m}_{i}^{\text {out }} h_{i}^{\text {out }}-\dot{m}_{i}^{\text {in }} h_{i}^{\text {in }} \\
\frac{d M_{i}}{d t} & =\dot{m}_{i}^{\text {in }}-\dot{m}_{i}^{\text {out }} \\
P_{i} V_{i} & =M_{i} R T_{i}
\end{aligned}
$$

where the rate at which work is done by the pressure on the boundaries is given by $P_{i} d V_{i} / d t$. The heat transfer across the boundaries of the $\mathrm{CVs}$ is given by:

$$
\begin{aligned}
& \dot{Q}_{i}=q^{\prime \prime} A_{i H T} \\
& q^{\prime \prime} \cong h_{H T}\left(T_{e n v}-\bar{T}_{H T}\right)
\end{aligned}
$$

Such a representation is chosen because the heat transfer out of a high temperature Roots compressor is dominated by the cooling conditions available on the outer surface of the blower unless a special internal cooling is involved. Therefore $h_{H T}$ is an overall transfer coefficient which includes conduction across of the casing, convection and radiation. $\bar{T}_{H T}$ corresponds to some average surface temperature of the unit. It is impossible to have an accurate prediction of the temperature distribution on a compressor unless a specific study is conducted. For the purposes of this paper $\bar{T}_{H T}$ is taken as following and should provide an adequate estimate:

$$
\bar{T}_{H T}=\sum A_{i H T} T_{i H T} / \sum A_{i H T}
$$

The other terms in the Equations (1)-(2) are:

$$
\begin{gathered}
U_{i}=C_{v} T_{i} \\
h_{i}^{\text {out }}=\left\{\begin{array}{ll}
C_{p} T_{1} & i=1 \\
0 & i=2, \\
C_{p} T_{3} & i=3
\end{array} \quad h_{i}^{\text {in }}= \begin{cases}0 & i=1 \\
C_{p} T_{1} & i=2 \\
0 & i=3\end{cases} \right.
\end{gathered}
$$




$$
\dot{m}_{i}^{\text {out }}=\left\{\begin{array}{ll}
\dot{m}_{e}+\dot{m}_{n}+\dot{m}_{l} & i=1 \\
0 & i=2, \\
\dot{m}_{e}+\dot{m}_{l} & i=3
\end{array} \quad \dot{m}_{i}^{\text {in }}= \begin{cases}0 & i=1 \\
\dot{m}_{n} & i=2 \\
0 & i=3\end{cases}\right.
$$

Equation (9) shows that the leakage is considered only for CV1 and CV3. Thus assessment of the leakage is approximate; a better model, which takes into consideration each leakage path and adjusts according to thermal expansion, could be included in future models. Presently, the leakage model is based on the corresponding total clearance area. This includes the flows in the passages located between: the casing and the impellers' tips; casing and lateral sides of the impellers (reduced by the shafts); and the two intermeshing impellers. The latter passage geometry is dependent on the current impeller position and widely varies with time for circular geometry.

The mass flow for choked flow through a nozzle configuration is obtained from:

$$
\begin{aligned}
\dot{m}_{S_{\text {choked }}} & =A_{s} P_{i}\left(\frac{k}{R \cdot T_{i}}\right)^{1 / 2}\left(\frac{2}{k+1}\right)^{\left(\frac{k+1}{2(k-1)}\right)} \\
& =\frac{A_{s} C_{v}^{1 / 2} M_{i} T_{i}^{1 / 2}}{V_{i}} f(k) \\
f(k) & \equiv k^{1 / 2}(k-1)^{1 / 2}\left(\frac{2}{k+1}\right)^{\left(\frac{k+1}{2(k-1)}\right)}
\end{aligned}
$$

Where $f(k)$ is a constant for a given gas; for air, $f(7 / 5)=$ 0.433 .

During the numerical simulation independent unit-step functions monitor when the pressure ratio between the communicating $\mathrm{CV}$ s or the environment and CVs falls to critical, and automatically switches to the non-choked flow expression

$$
\begin{aligned}
\dot{m}_{s} & =A_{s}\left(\frac{2 k}{k-1} P_{i} \rho_{i}\left(\frac{P_{j}}{P_{i}}\right)^{\frac{2}{k}}\left(1-\left(\frac{P_{j}}{P_{i}}\right)^{\frac{k-1}{k}}\right)\right)^{1 / 2} \\
& =\dot{m}_{s_{\text {chooked }}} g\left(k, \frac{P_{j}}{P_{i}}\right) \\
g\left(k, \frac{P_{j}}{P_{i}}\right) & =\left(\frac{2}{k-1}\left(\frac{k+1}{2}\right)^{\frac{k+1}{k-1}}\left(\frac{P_{j}}{P_{i}}\right)^{\frac{2}{k}}\left(1-\left(\frac{P_{j}}{P_{i}}\right)^{\frac{k-1}{k}}\right)\right)^{1 / 2}
\end{aligned}
$$

where $g\left(k, P_{j} / P_{i}\right)$ is a non-dimensional function, which represents the flow rate adjustment from the choked regime to the non-choked regime. It is a function of the specific heat ratio and pressure ratio between the two regions. As pressure equilibrates $g$ decreases such that:

$$
\begin{gathered}
\lim \left(g\left(k, P_{j} / P_{i}\right)\right)=0 \\
P_{j} / P_{i} \rightarrow 1
\end{gathered}
$$

Equations (1), (2) and (10) can be rewritten in a nondimensional form:

$$
\begin{aligned}
\frac{d \mathbf{T}_{i}}{d \mathbf{t}}+\frac{(k-1)}{\mathbf{V}_{i}} \frac{d \mathbf{V}_{i}}{d \mathbf{t}} \mathbf{T}_{i} & +(k-1) \dot{\mathbf{m}}_{i}^{\text {out }} \frac{\mathbf{T}_{i}}{\mathbf{M}_{i}} \\
+\dot{\mathbf{m}}_{i}^{\text {in }} \frac{\left(\mathbf{T}_{i}-k \mathbf{T}_{1}\right)}{\mathbf{M}_{i}} & =\frac{b \mathbf{A}_{i H T}}{\mathbf{M}_{i}} \\
\frac{d \mathbf{M}_{i}}{d \mathbf{t}} & =\dot{\mathbf{m}}_{i}^{\text {in }}-\dot{\mathbf{m}}_{i}^{\text {out }} \\
\dot{\mathbf{m}}_{s_{\text {choked }}} & =a \frac{\mathbf{A}_{s} \mathbf{M}_{i} \mathbf{T}_{i}^{1 / 2}}{\mathbf{V}_{i}}
\end{aligned}
$$

where

$$
\begin{array}{lll}
\mathbf{t} \equiv t / t_{c}, & \mathbf{T}_{i}(\mathbf{t}) \equiv T_{i}(t) / T_{2}(0), & \mathbf{M}_{i}(\mathbf{t}) \equiv M_{i}(t) / M_{2}(0), \\
& \mathbf{V}_{i}(\mathbf{t}) \equiv V_{i}(t) / V_{2}(0), & \mathbf{A}_{\text {any }}(\mathbf{t}) \equiv A_{\text {any }}(t) / A_{e}
\end{array}
$$

and

$$
\begin{aligned}
a & \equiv f(k) A_{e}\left[C_{v}^{1 / 2} T_{2}^{1 / 2}(0)\right] /\left[V_{2}(0) / t_{c}\right] \\
b & \equiv\left[q^{\prime \prime} A_{e} \cdot t_{c}\right] /\left[C_{v} m_{2}(0) T_{2}(0)\right] \\
& =(k-1)\left[q^{\prime \prime} A_{e}\right] /\left[P_{2} V_{2}(0) / t_{c}\right]
\end{aligned}
$$

Temperature, mass and volume in the equations above are non-dimensionalized by the conditions in the well immediately after it separates from the inlet region. Instead of introducing a third control volume for the inlet, this approach allows to simplify referencing to the conditions of the incoming gas. Assuming that mixing of the leaking and incoming gases takes place adiabatically at the inlet pressure, the inlet temperature and required new mass are recalculated based on conservation of mass and energy. The leaked mass and energy are obtained from the solution by integrating the corresponding terms.

All areas in the model are non-dimensionalized with respect to the exit area. The volume of the well also has an important role because it is directly related to the theoretical compressor displacement volume $V_{D}=2 N \cdot V_{2}(0)$. For time, $t_{c}(\mathrm{sec})=$ $60 /(2 N \cdot r p m)$ the time of compression is used, which ties together such important model parameters as rotational speed and number of lobes. Thus the term $\left[V_{2}(0) / t_{c}\right]$ in Equation (17) equals $\dot{V}_{D}$, the compressor geometric volume flow rate.

The well, reservoir and combined volumes are assumed to vary linearly with time. Our analysis of the circular lobe geometry, as well as the analysis of (Patterson, 1960), indicates that the variation of the working volumes with angle of rotation is linear to within a few percent. For three- and two-lobe circular impellers, the deviation from linear is only slightly more than for four-lobe impellers. The larger the reservoir volume the better the linearity assumption becomes. The reservoir volume is taken to be $\mathbf{V}_{\mathbf{1}}(\mathbf{t})=\mathbf{r}-\mathbf{t}$, where $\mathbf{r}=V_{1}(0) / V_{2}(0)$. The normalized well volume is assumed to be unity throughout the expansion-mixing and compression stages. The combined volume is a simple superposition of the two at the start of the compression phase.

The dimensionless equations above allow the analysis of a general compressor. The dimensionless coefficient $a$, Equation 
(17), will be called a compression coefficient. It defines the level of compression achieved in a Root's type device. The lower the value of $a$, the higher the final pressure ratio obtained. On the one hand, $a$ is independent of the number of the lobes and thus equally models different configurations of the device. On one hand, it incorporates diverse effects from different aspects of the compressor. For example, an increase in leakage would result in a square root increase of $a$ through the initial temperature in the well, but can be adjusted back by a linear decrease of the outlet area or an increase in the rotational speed. The kind of interplay of the variables in $a$ outlined above provides a valuable tool in the design process. There exists an upper value of $a$ at which compression can be sustained; for example, due to a very large exit area.

Heat transfer in the model is introduced through a dimensionless thermal transport coefficient $b$. Its nature is similar to $a$ and provides an estimate of the combined effect of several design parameters on the heat transfer in the compressor. It is most likely that the design of an MRH will require some heat removal $q^{\prime \prime}<0$; for a device which is insulated and is approximately adiabatic, $q^{\prime \prime}=0$.

\section{DISCUSSION OF THE NOZZLE}

The well filling process as we identified above is a complex, multi-stage process. Two control volumes become connected by an "expansion nozzle," which is naturally formed by an impeller tip and the compressor casing. In a high temperature Root's compressor the pressure ratio is sufficiently large for a choked flow to develop at the beginning. The present model is only concerned with the mass and energy of the high temperature gas flowing into the well. This is adequately modeled by Equation (16). The shape of the "nozzle" can be described as a converging-diverging nozzle with time-varying geometry. As long as the flow through the "nozzle" is choked, the throat area is used in the calculation, Figure 2. However, as the pressure in the throat rises to the critical pressure

$$
\frac{P_{t}^{*}}{P_{1}}=\left(\frac{2}{k+1}\right)^{k /(k-1)}
$$

and above Equation (16) is replaced by Equation (12) in nondimensional form and the flow rate becomes a function of the pressure in the well (which is not the same as the throat pressure). The exit area of the "nozzle" should be taken at the cross section which is at well pressure. This choice is somewhat arbitrary. For example, a location where secondary flows, generated due to the nozzle's geometrical expansion, are avoided. The angle $\delta$, at which such flows are prevented from forming, is about 7 to 10 degrees, Figure 3 . Thus, if there are two pressure ratios $P_{1} / P_{2}$ and $P_{t} / P_{2}$ available for calculation, $P_{t} / P_{1}$ is implicitly evaluated and checked against the critical condition, Equation (19). If the pressure is above critical then Equation (12) is applied.

Next is an example analysis of such a particular configuration. This analysis makes use of a simplified geometry. If a more

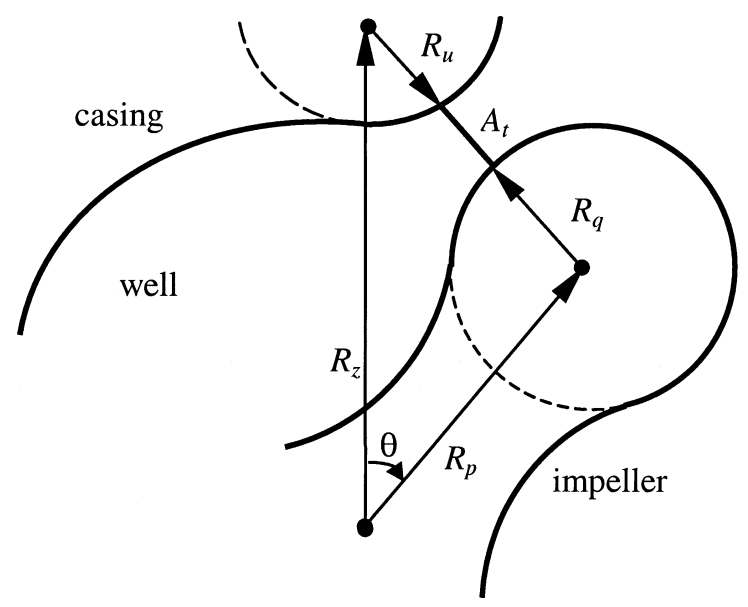

FIGURE 2

Shows the throat of the "nozzle" formed by the upper casing and the impeller during the expansion-mixing process. The dashed line shows the circular geometry of the example.

complex configuration is involved, a numerical approach could be applied. The impeller lobe considered, the inner surface of the casing and reservoir after the breakaway point all have a circular geometry, Figure 2. Since the throat is formed by two circles, the minimum distance (the throat) is a distance between the centers of the circles, Equation (20), with the radii subtracted, Equation (21):

$$
\begin{aligned}
& \left\{\begin{array}{l}
x_{u}=0 \\
y_{u}=R_{z}
\end{array}, \quad\left\{\begin{array}{l}
x_{q}=R_{p} \sin \theta \\
y_{q}=R_{p} \cos \theta
\end{array}\right.\right. \\
& A_{t} / W=\left(\left(R_{z}-R_{p} \cos \theta\right)^{2}\right. \\
& \left.+\left(R_{p} \sin \theta\right)^{2}\right)^{1 / 2}-R_{u}-R_{q}
\end{aligned}
$$

where $R_{z}=R_{p}+R_{q}+R_{u}+$ clrn.

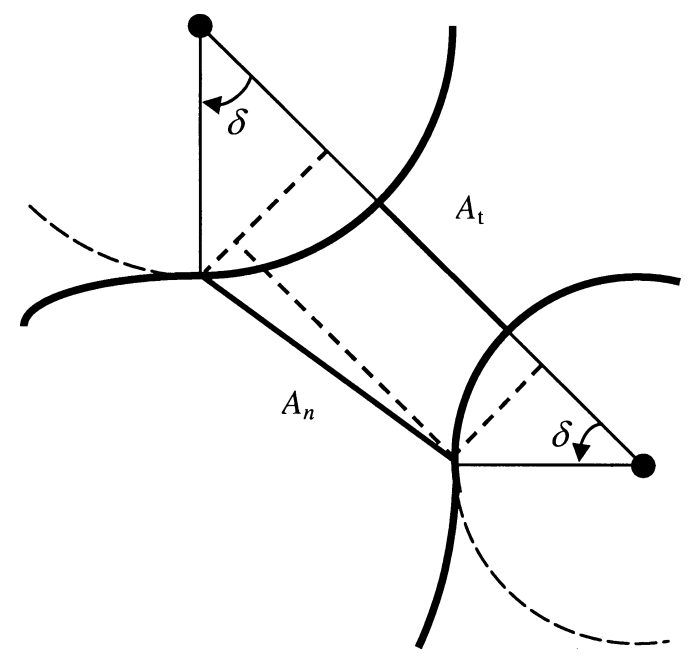

FIGURE 3

Shows the generation of $A_{n}$ between the casing and the impeller. The dashed lines represent corroborative geometry. 
Imposing the angle $\delta$ on the present geometry, Figure 3, generates the nozzle area $A_{n}$ :

$$
\begin{aligned}
\left(A_{n} / W\right)^{2}= & \left(A_{t}+\left(R_{u}+R_{q}\right)(1-\cos \delta)\right)^{2} \\
& +\left(\left(R_{u}-R_{q}\right) \sin \delta\right)^{2}
\end{aligned}
$$

The variation of $P_{t} / P_{2}$ with the area ratio is then given by:

$$
\frac{A_{n}}{A_{t}}=\left(\frac{P_{t}}{P_{2}}\right)^{\frac{1}{k}}\left(\left(1-\left(\frac{P_{t}}{P_{1}}\right)^{\frac{k-1}{k}}\right) /\left(1-\left(\frac{P_{2}}{P_{1}}\right)^{\frac{k-1}{k}}\right)\right)^{1 / 2}
$$

All the required areas and pressures have been identified. The mass and energy flowing into the well can then be calculated throughout the expansion-mixing process for all possible flow regimes.

\section{NUMERICAL SIMULATION AND EXPERIMENTAL RESULTS}

A generic two lobe Root's compressor operating on air was chosen for this numerical simulation. Due to the cyclic nature of the compression in a Root's blower, the numerical integration of the equations was performed until the thermodynamic properties in the control volumes converged. The initial states for the integration are unity for the well conditions and an intelligently estimated state for the reservoir. Converged results for the no leakage condition are used as a starting point for the simulations having leakage flow and heat transfer.

Figure 4 shows the effect, which the ratio of well to reservoir volumes has on the pressure fluctuation in the reservoir during a compression cycle. For $\mathbf{r}=10$ the fluctuation is about $5 \%$ as opposed to $17 \%$ for $\mathbf{r}=2$. Although the maximum achieved pressure will be less, enlarging the reservoir volume can attain a significant reduction in noise. Size versus noise is a very important issue in automotive superchargers. On the other hand, $r$ has only small effect on the overall temperature versus pressure

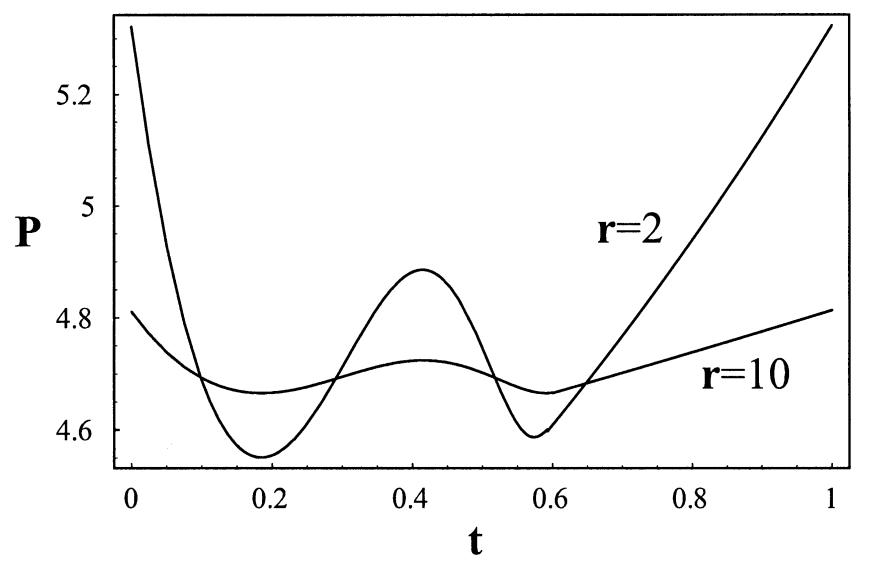

FIGURE 4

Pressure fluctuation in the reservoir as a function of its size,

$$
a=0.3 \text {. }
$$

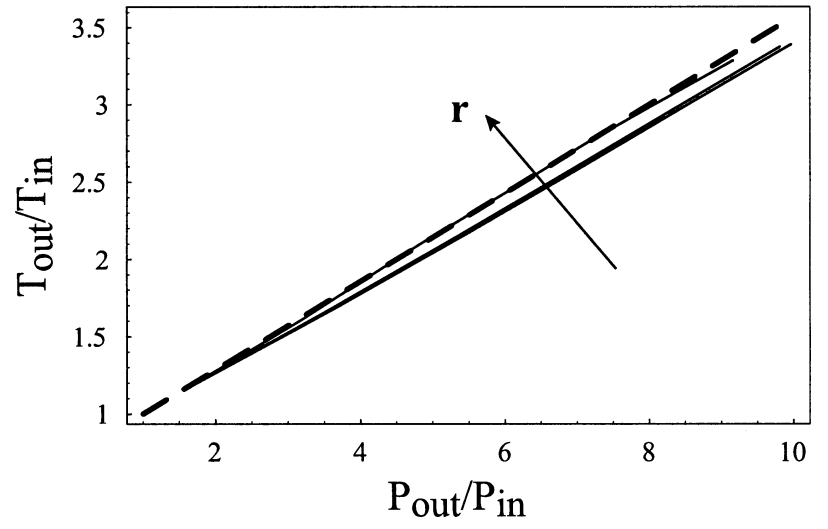

FIGURE 5

Effect of the reservoir volume $\mathbf{r}(1.5,2$, and 10) on the blower performance. Dashed line represents Kestin-Owczarek model $\mathbf{r} \rightarrow \infty$.

performance of the compressor, Figure 5. The model also shows a good agreement with the (Kestin and Owczarek, 1952) model developed for no leakage or heat transfer case.

Figure 6 represents signatures of both pressure in the well and reservoir. The mixing-expansion process takes place until both pressures equilibrate. The figure shows that the mixing time increases as does the pressure ratio following the reduction in the compression coefficient $a$. However, the increase in the mixing time is not as fast after the half time even for very high pressure ratios because the throat area, which is opening slowly in the first quarter of the compression cycle, becomes wide open by the half time providing an adequate mass flow to equilibrate the pressures in the control volumes.

One of the most important factors in operating a Root's type device is leakage. Figure 7 shows how fast the final temperature rises due to leakage. There are two important effects present. First, the device's mass throughput is decreased by the amount of mass leaked to the low pressure side. Second,

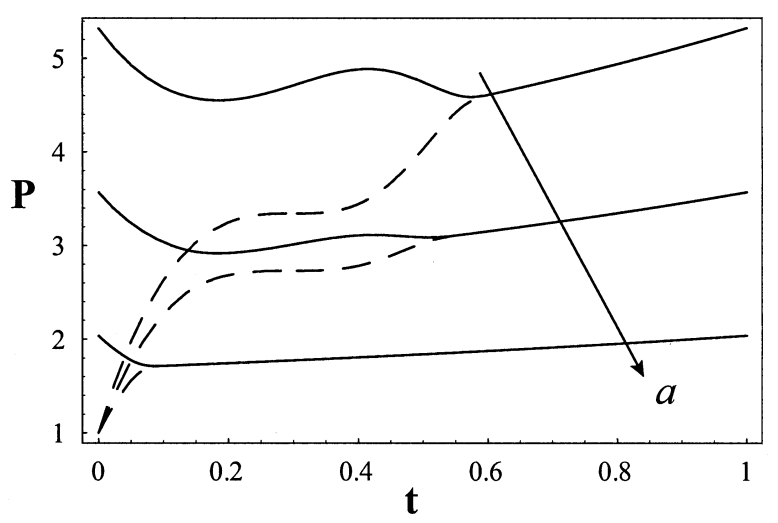

FIGURE 6

Effect of the compression coefficient $a(0.3,0.5$, and 0.7$)$ on the pressure in the control volumes. The solid line corresponds to the reservoir and dashed to the well, $\mathbf{r}=2$. 


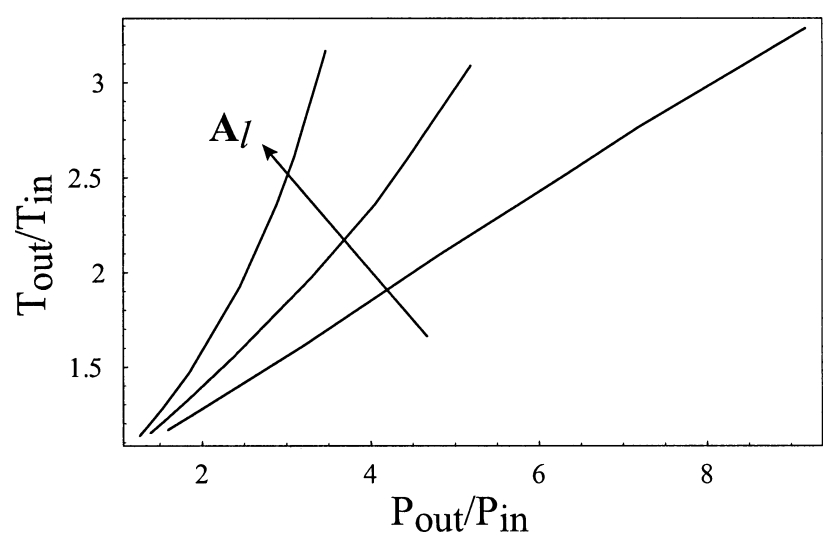

FIGURE 7

Effect of leakage $\mathbf{A}_{l}(0,0.2$, and 0.4), $\mathbf{r}=10$.

compression takes place at an elevated temperature resulting in "multi-recompression" and consequently very high output temperature even at relatively low pressure ratios.

The heat transfer in the system is another important component of the model. It is represented by the areas of heat transfer on the casing surface and $b$. Its effect, see Figure 8, is more straightforward than that of the leakage. The final temperature can be affected by as much as $20-30 \%$ at high temperatures, even in the case of natural convection and radiation at room conditions.

Figure 9 shows a very good agreement between the present model and the previously reported experiment (Blekhman et al., 2000 ) in capturing the reservoir pressure signature of a Weiand Pro-Street 142 automotive supercharger at $8900 \mathrm{rpm}$. The presented normalized results indicate the model's capabilities in capturing the gas-dynamic behavior. There are two "blips" in the data and one in the model. The first "blip," initially believed to be a product of shockwave interactions, can be mainly related to the ability of the reservoir to expel mass through the exit area and the "nozzle" while undergoing reduction in the volume dur-

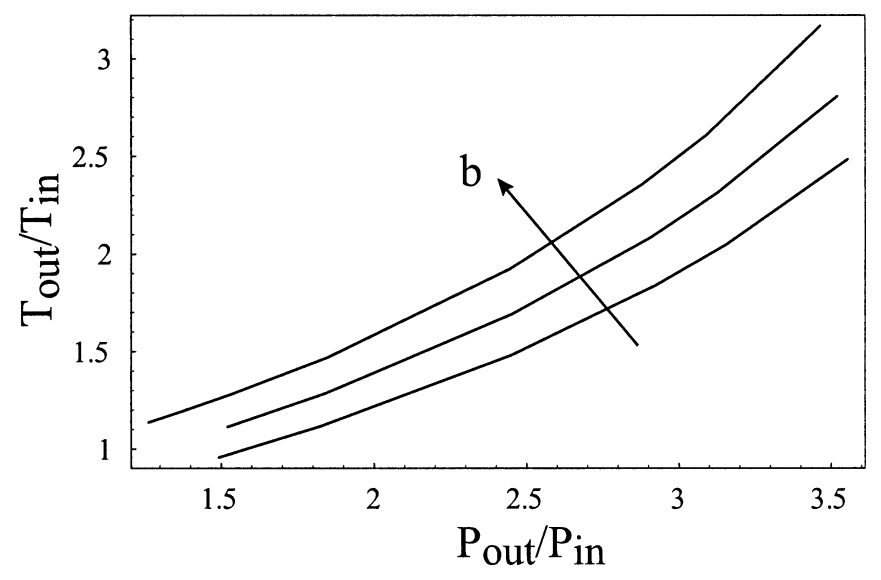

FIGURE 8

Effect of heat transfer, $b=(0,-5 \mathrm{E}-5,-10 \mathrm{E}-5)$, $\mathbf{A}_{l}=0.4, \Sigma \mathbf{A}_{H T}=2300, \mathbf{r}=10$.

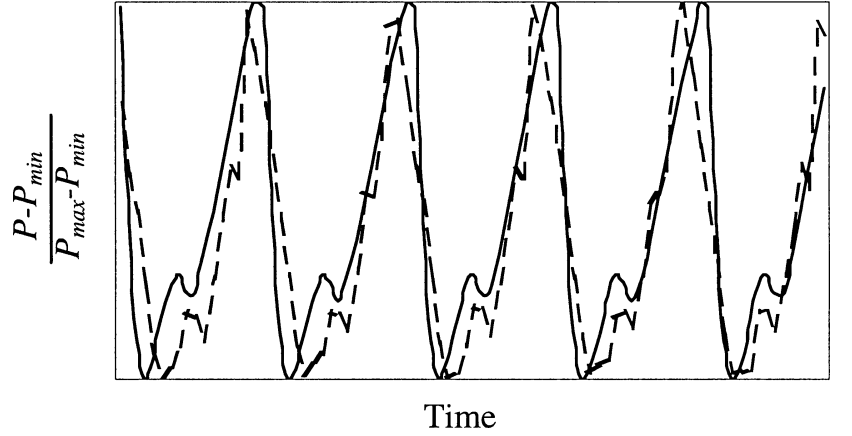

FIGURE 9

Comparison of the normalized pressure signature in the reservoir. Solid line-model, dashed-experiment.

ing the expansion-mixing stage. The "blip" magnitude and the time of occurrence are dependent on a combination of several factors, such as $\mathbf{r}, a$ and the "nozzle" enlargement rate, as can be seen from Figures 4 and 6 . The second "blip," occurring during the compression stage and not reproduced by the theory, might be attributed to the peculiarities of the supercharger geometry not reflected in the current model.

A more complex series of experiments was conducted using a Dresser-Roots Whispair RAM 404 blower. A maximum steady state outlet temperature of $650 \mathrm{~K}$ was achieved at a pressure ratio of 3.2 and speed of $2180 \mathrm{rpm}$. The Whispair design has a proprietary feature: an enlarged reservoir with slots, which alternatively open to the wells allowing for gas in the reservoir bleed into the normally low pressure wells. According to the manufacturer this allows for slower pressure build up in the exposed well, and, consequently, for reduction in pressure fluctuation and overall noise produced. In order to accommodate this feature, Equations (22)-(23) were significantly modified. The compression in these experiments was achieved by drawing a vacuum at the inlet; therefore, another

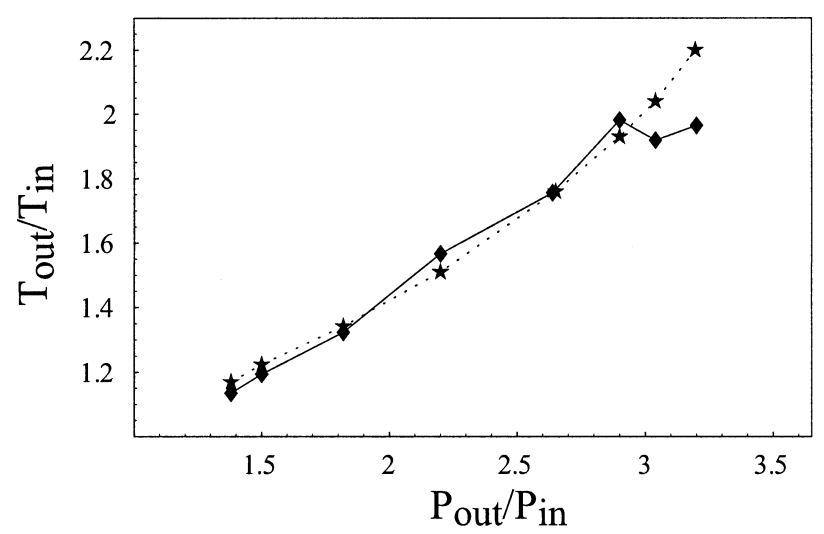

FIGURE 10

Comparison of calculated and measured performance for Dresser-Roots Whispair RAM 404 blower. Solid line-model, dashed-experiment. 
modification was introduced to compensate for this in the compression model.

Measured heat transfer and leakage rates were used as input to the current calculation rather than predicted. All these modifications and the details of the experiment will be described in a paper to be written. Figure 10 shows that the proposed model demonstrates very good agreement with the experiment. The deviation in the high pressure region can be attributed to the uncertainty of the leakage under extremely low flow rates through the blower at these regimes.

\section{CONCLUSIONS}

The model presented here provides qualitative and quantitative analysis of a high pressure, high-temperature Root's type device as well as guidance for future designs of an MRH. The model modified to two control volumes adequately describes the operational parameters. Our model has extended the ability to predict the temperature increase to higher pressure ratios. Good agreement with the experimental data has been shown for pressure ratios up to three.

Close attention should be paid to the modeling of the "nozzle" in order to have accurate analysis of the expansion-mixing process and assessment of the noise contributing factors.

The model contains dimensionless compression and thermal transport coefficients which permit a design/performance tradeoff assessment in terms of: gas used, exit area, operating speed, machine size etc. Modeling of leakage and heat transfer may be improved by introducing more detailed models of each.

\section{NOMENCLATURE}

\section{Notation}

\section{A, A}

$a$

$b$

clrn

$C_{p}, C_{v}$

$\mathrm{CV}$

$\delta$

$f$

$g$

$h$

$k$

$M, \mathbf{M}$

$\dot{m}, \mathbf{m}$

$N$

$P, \mathbf{P}$

$\dot{Q}$

$q^{\prime \prime}$

$R$

$\mathbf{r}$ area, dimensionless area

dimensionless compression coefficient, Equation (17)

dimensionless thermal transport coefficient, Equation (18)

clearance

specific heats

control volume

angle at which secondary flows are avoided

dimensionless coefficient, Equation (11)

Conversion coefficient to subsonic flow, Equation

(13)

specific enthalpy, overall transfer coefficient

specific heats ratio

mass of a control volume, dimensionless mass

mass flow rate, dimensionless flow rate

number of lobes per impeller

pressure, dimensionless pressure

heat transfer rate

heat flux

gas constant for gas or radius

well to reservoir volumetric ratio at time zero rpm

$T, \mathbf{T}$ and $\bar{T}$ temperature, dimensionless and average temperature

$t, t_{c}$ and $\mathbf{t}$ time, cycle and dimensionless time

$\theta \quad$ impeller rotation angle

$U \quad$ internal energy

$V, \mathbf{V} \quad$ volume, dimensionless volume

$W \quad$ impeller length

$x, y \quad x$ and $y$ coordinates of a circle center

$\begin{array}{ll}\text { Superscripts and Subscripts } \\ 1 & \text { reservoir conditions } \\ 2 & \text { well conditions } \\ 3 & \text { combined volume conditions } \\ i, j & \text { any of the } 1,2, \text { and } 3 \\ \text { in } & \text { inlet conditions, or into a control volume } \\ \text { out } & \text { reservoir conditions at the beginning of the cycle, } \\ & \text { or out of a control volume } \\ H T & \text { heat transfer on the casing surface } \\ e & \text { exit, out of the reservoir } \\ n & \text { nozzle formed between the well and reservoir } \\ l & \text { leakage } \\ e n v & \text { environment } \\ s & \text { any of the } e, n, l \\ t & \text { throat } \\ \text { any } & \text { any of the } e, n, l \text { and } H T \\ p & \text { pitch } \\ q & \text { lobe upper circle } \\ u & \text { casing geometry connecting the well and reservoir } \\ z & \text { combined distance } \\ \text { min, max } & \text { minimum, maximum } \\ * & \text { critical condition }\end{array}$

\section{REFERENCES}

Blekhman, D. I., Mollendorf, J. C., Felske, J. D., and Lordi, J. A. 2000. Analysis of high-pressure-ratio root's type compressor producing a very high-temperature outlet gas stream. Proceedings of the 8th International Symposium on Transport Phenomena and Dynamics of Rotating Machinery, Honolulu, Hawaii, 26-30 March.

Dubrin, J. W., and Harri, J. G. 1979. A mechanical heater for producing high-temperature gas streams. Energy Research LLNL: $18-25$.

Gardiner, A. W. 1926. A roots-type aircraft engine supercharger. J SAE 19:253-264.

Kestin, J., and Owczarek, J. A. 1952. The expression for work in a roots blower. Inst. Mech. Engrs. Proc. 1b:9198.

Merala, R., Hubbard, M., and Miyano, T. 1988. Modeling and simulation of a supercharger, dynamic systems, measurement and control. Trans. ASME 110:316-323.

Patterson, J. 1960. Theoretical aspects of the roots air motor. Mech. Engrng. Sci. 2:227-241.

Patterson, J., and Ritchie, J. B. 1969. Roots blower performance. Int. J. Mech. Sci. 11:575-593. 
Russo, A. L. 1999. Private communication [see also, Recompression heater failure: Dynamic analysis of rotating train, Pretech Inc, Pleasant Hill, CA].

Russo, A. L., and Daiber, J. W. 1976. A gas dynamic device for the production of a uranium atom flux for isotope separation. ERDA Report April.

Smith, H. 2001. A theoretical consideration of gas dynamic processes for ethylene production, Thesis, SUNY Buffalo.

Sorenson, S. C. 1984. Simulation of a positive displacement supercharger. SAE 840244, 115-129.

Taylor, C. 1966. The internal-combustion engine in theory and practice, Cambridge, MA, M. I. T. Press.
Weatherston, R. C., Russo, A. L., Smith W. E., and Marrone, P. V. 1959. Gas dynamics of a wave superheater facility for hypersonic research and development. AFOSR TN59-107.

Weatherston, R. C. 1969. The multi-recompression heater, a new concept for large scale hypersonic testing. AIAA 4th Aerodynamic Testing Conference, Paper No. 69-332, Cincinnati, April.

Ucer, A. S., and Celic, I. 1980. Analysis of flow through roots blower systems. Proceedings of the Purdue Compressor Technology Conference, West Lafayette, Indiana, July 23-25.

Uthoff, L. H., and Yakimow, J. W. 1988. Development of the eaton supercharger, SAE 870355, 5.474-5.483. 

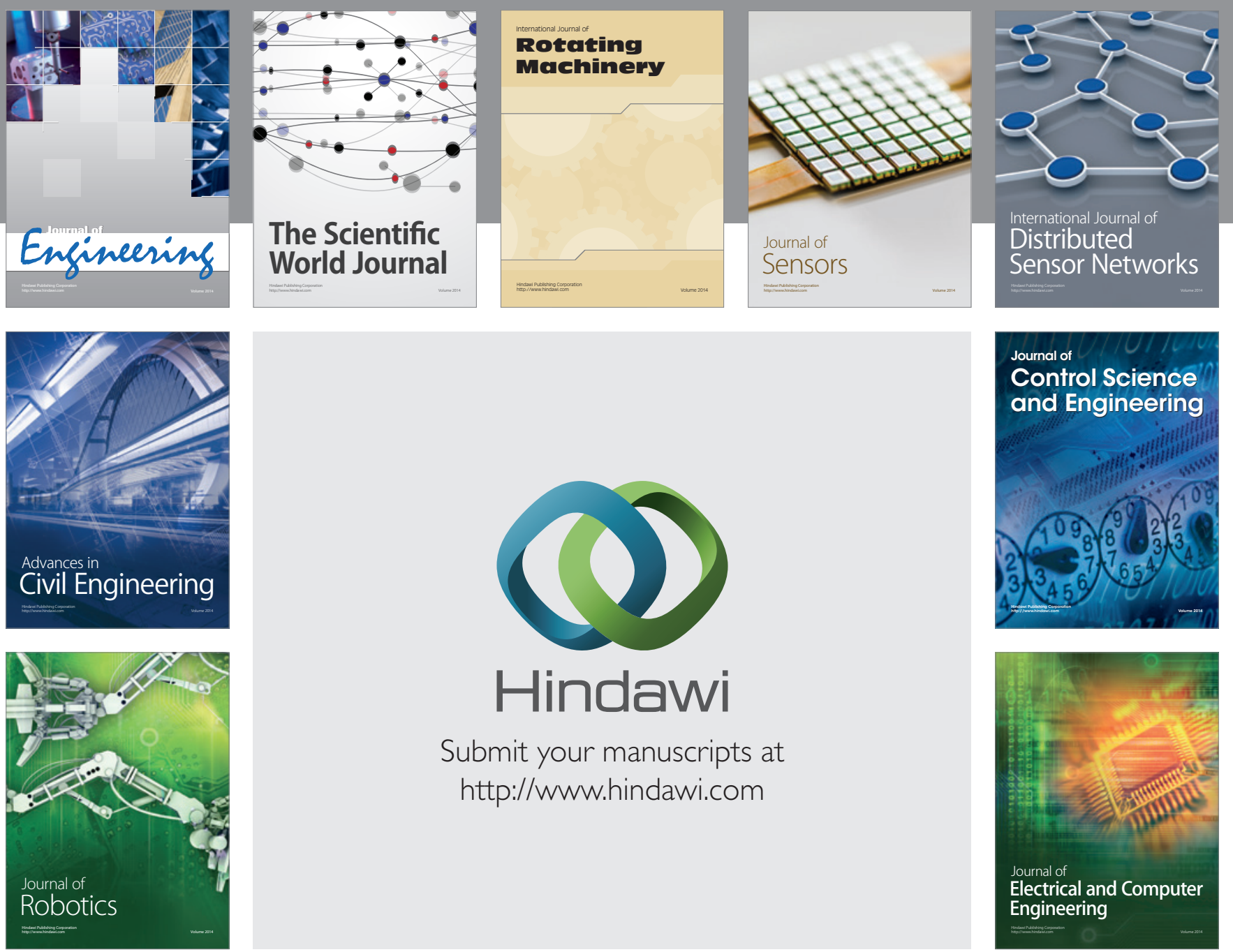

Submit your manuscripts at

http://www.hindawi.com
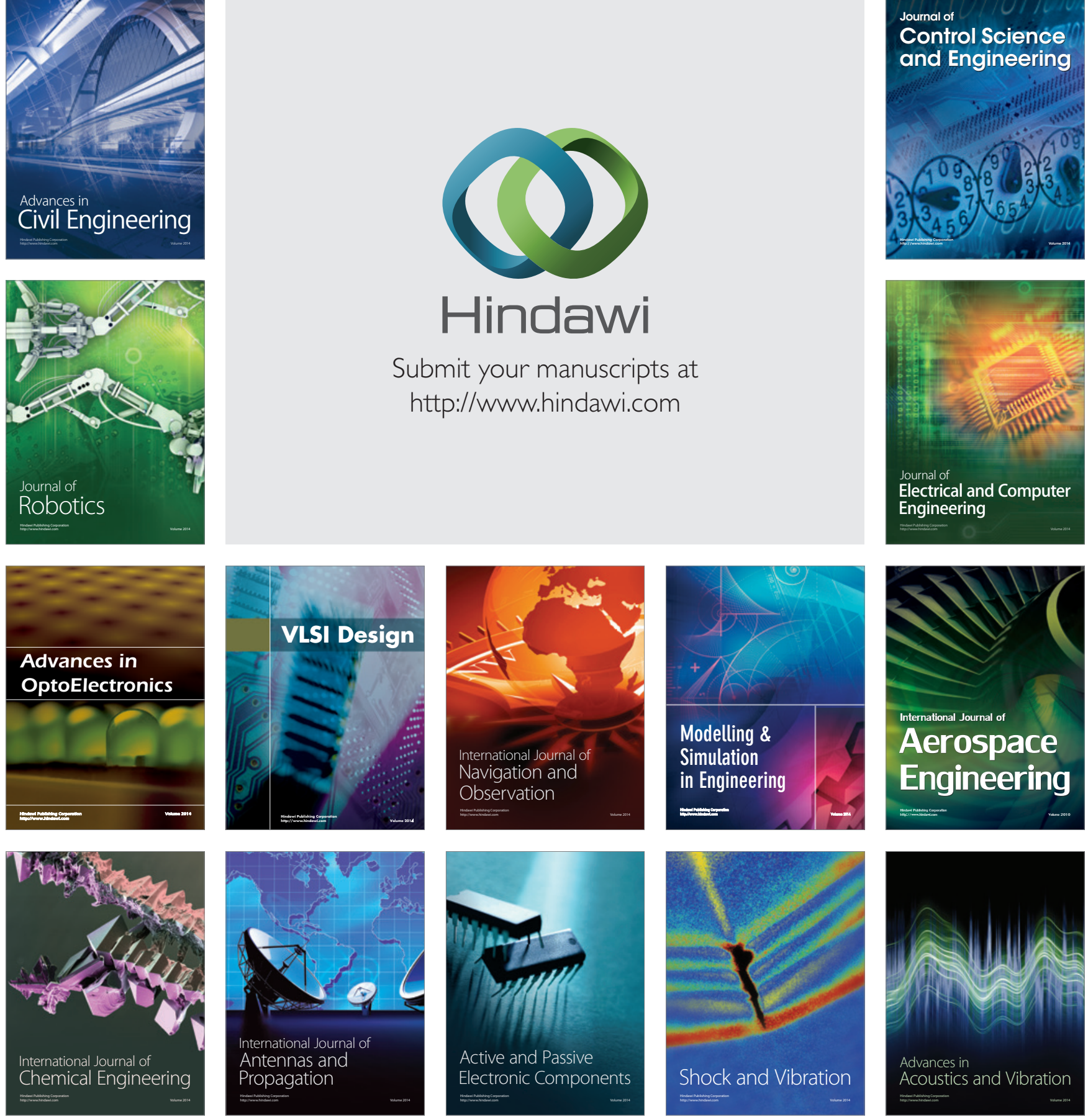